\title{
Degenerate Convex Hulls On-Line in Any Fixed Dimension
}

\author{
H. Brönnimann \\ INRIA Sophia-Antipolis, 2004 Route des Lucioles, B.P. 93, \\ 06902 Sophia-Antipolis Cedex, France \\ Herve.Bronnimann@sophia.inria.fr
}

\begin{abstract}
Randomized algorithms have, since the pioneering work of Clarkson and Shor, occupied the front stage of computational geometry. Yet despite their simplicity, they often cannot handle or be extended to cope with degenerate cases. The main goal of this paper is to show how, for the special case of convex hulls, a simple modification of the formulation of an on-line algorithm by Boissonnat et al., based on that of Clarkson and Shor, can handle the case of degenerate sets of points for computing convex hulls on-line in $\mathbb{R}^{d}$, for a fixed $d \geq 2$. At the same time, a standard randomized analysis allows us to prove the expected time complexity to be within $O\left(n \log n+n^{\lfloor k / 2\rfloor}\right)$, where $k$ is the dimension of the convex hull. The latter bound is always $O\left(n \log n+n^{\lfloor d / 2\rfloor}\right)$. The analysis uses connections between regions, pivots, flags, and barycentric triangulations.
\end{abstract}

\section{Introduction}

Given $n$ points in $\mathbb{R}^{d}$, to enumerate the facets of their convex hull is a classical problem of optimization and computational geometry [1], [2], [5], [12], [8], [15], [17], [20], [21]. The scenario in which we work here is that $d$ is constant and small (say, $d \leq 10$ ), but the number of points is large, they are inserted on-line, and they may not be in general position. Moreover, we not only produce the facets of the convex hull, but the whole face lattice.

Several algorithms have been given in various cases. In the off-line case, the points can be sorted and their affine span computed. Then the algorithms of [2], [8], [9], and [15] can all be applied. The optimal deterministic of Chazelle [9] assumes general position. The randomized incremental algorithms that use a conflict graph [12] or an influence graph [4], [5] solve a related problem which is only equivalent to the convex hull problem for nondegenerate instances. In fact these algorithms, which are worst-case optimal for nondegenerate inputs, could perform badly (up to quadratic slowdown) when many points belong to a facet. We show a sample of the problems that arise with degenerate inputs and on-line algorithms in Section 2.2. 
The problems of degeneracies is well known and several approaches are possible. Perturbation methods (e.g., [15]) remove degeneracies but the problem solved is not the original problem. This can be an issue when counting facets and not simply computing a volume. The necessary postprocessing is often not detailed in the literature (one notable exception is Emiris' implementation [16]). If we insist on solving the original problem, the off-line algorithms can usually be adapted easily [13], [15], [18]. To solve the on-line case, the only algorithm available in any dimension is given by Burnikel et al. [7]. Although it is a fairly small modification of an algorithm that works on nondegenerate inputs [11], their analysis is involved and they do not provide the same upper bounds for degenerate and nondegenerate cases. In particular, it is not known if their algorithm runs in expected time $O\left(n \log n+n^{\lfloor d / 2\rfloor}\right)$ for fixed $d$ on all instances. As they put it, in degenerate cases the "running time depends also on the structural properties of lower-dimensional faces," which then becomes hard to analyze.

We propose an extension to the algorithms of [4] and [5] which is on-line, can handle arbitrarily degenerate instances, and is fairly easy to analyze thanks to its connections to flag vectors [3]. Its expected running time is $O\left(n \log n+n^{\lfloor d / 2\rfloor}\right)$ on all instances. The same solution was proposed for dimension 3 only by Matoušek et al. [13, pp. 205 and 242]. Since the problem is usually the space needed by the data structure, we propose a version of our algorithm which requires less storage, by using a persistent data structure [14], [19]. This data structure can be abbreviated further by the techniques of [21]. Interestingly, the bounds we give can be used to analyze many pivoting algorithms in degenerate cases, such as that of [18].

We organize the paper as follows. Section 2 gives our formulation of the problem, and our extension to the framework of [4] and [5], and in Section 3 we devise a persistent data structure for storing the incidence lattice of a polytope which implicitly contains the structures of Section 4. In Section 4 we analyze the various triangulation schemes (which may be of independent interest) and complete the proof of complexity of the persistent graph. We conclude on the limitations and questions raised by our approach.

\section{A Formalism for Computing Convex Hulls}

We assume that the reader is familiar with the notions of polytope, faces, incidence of faces, and simplicial complex [5], [15], [17]. For a fixed dimension $d$, the upper-bound theorem states that a $d$-polytope with $n$ vertices or $n$ facets has $O\left(n^{\lfloor d / 2\rfloor}\right)$ faces and pairs of incident faces. The problem we address here is that of computing the whole incidence graph of $\operatorname{conv}(\mathcal{S})$ for a set $\mathcal{S}$ of $n$ points in $\mathbb{R}^{d}$. The problem here is presented in primal space (even though Clarkson and Shor's algorithm is usually presented in a dual-based setting).

Some familiarity with incremental randomized algorithms [12], [5] is assumed in this section. In this framework, objects are inserted from a universe, they define a set of regions, and the set of regions which do not conflict with the objects (the free regions) is maintained. The incremental algorithm maintains the set of free regions in a data structure, a conflict graph [12] or an influence graph [4], [5]. To have an on-line algorithm, we work with an influence graph. 


\subsection{An Overview of the Influence Graph}

The influence graph (as described in [5]) is a structure that stores the history of the incremental construction and depends on the order in which the objects have been processed by the algorithm. This graph represents the regions created by the algorithm during the incremental construction, and can be used to detect the conflicts between these regions and a new object. When the algorithm uses a conflict graph, the set of data is known in advance, and the algorithm may then compute the objects in $\mathcal{S}$ that conflict with a given region. However, an on-line algorithm does not assume any knowledge of the objects to be processed. Thus it must be able to describe the entire domain of influence of a region, which is the subset of all the objects in the universe that conflict with this region.

The influence graph is a directed, acyclic, and connected graph. It possesses a single root, and its nodes correspond to the regions created by the algorithm during its entire history. Therefore, a node corresponds to a region that was free at some point during the execution of the algorithm. A leaf corresponds to a region which is free after the last insertion. Two very important properties are required: (1) the number of children of each node of the influence graph is bounded by a constant, and (2) the domain of influence of a region associated with a node of the influence graph is contained in the union of the domains of influence of the regions associated with the parents of that node (the inclusion property).

An on-line algorithm will use the influence graph to maintain the set of free regions (stored at the leaves) under insertion of an object $O$ in two phases: In the locating phase it will traverse the graph recursively by visiting all the nodes that conflict with $O$. Then the leaves which conflict with $O$ are said to be killed while $O$ defines some new regions which are said to be created during this incremental step. In the update phase the algorithm modifies the influence graph accordingly.

Assume that an algorithm satisfies the update conditions: the time to compute a conflict is constant, the parents of a node created by an object $O$ are nodes that are killed by $O$, and updating the influence graph takes time linear in the number of nodes killed or created at each step. The first condition is not essential: if a conflict takes a certain time to be computed, the running time can be multiplied by this amount. The second ensures that all the free regions that conflict with the new point are all retrieved in the location phase. The last allows us to view the update phase as a black box and still analyze the algorithm.

Let $f_{0}(r, \mathcal{S})$ be the expected number of free regions of a random sample of $r$ objects among $\mathcal{S}$. The theorem of the influence graph [4], [5] states that, if the update conditions are satisfied, the expected storage and time used by the algorithm to process $n$ objects are respectively

$$
O\left(\sum_{r=1}^{n} \frac{f_{0}(r, \mathcal{S})}{r}\right), \quad \text { and } \quad O\left(n \sum_{r=1}^{n} \frac{f_{0}(r, \mathcal{S})}{r^{2}}\right) .
$$

\subsection{Its Application to Convex Hulls}

For convex hulls, the universe is $\mathbb{R}^{d}$, the objects are the input points. A region defined by $\left(p_{0}, \ldots, p_{d}\right)$ is the union of the two open half-spaces bounded by $\left(p_{0}, \ldots, p_{d-1}\right)$ and 
by $\left(p_{1}, \ldots, p_{d}\right)$ that do not contain $p_{0}, \ldots, p_{d}$. The free regions over a set $\mathcal{S}$ of $n$ points represent exactly the $(d-2)$-dimensional facets (also called the ridges) of the convex hull of $\mathcal{S}$, and encode the adjacency relationship between its two incident facets. Thus from the free regions, one can easily recover the adjacency graph of facets of $\operatorname{conv}(\mathcal{S})$. The upper-bound theorem assures us that there are at most $O\left(n^{\lfloor d / 2\rfloor}\right)$ free regions defined by the objects in $\mathcal{S}$.

An algorithm that maintains those regions, and therefore the ridges of the convex hull, is described in [5], and uses the influence graph as outlined above. In particular, the number of regions defined by a sample of $r$ objects is $f_{0}(r, \mathcal{S})=O\left(r^{\lfloor d / 2\rfloor}\right)$. The theorem of the influence graph states that the expected time complexity is $O(n \log n+$ $n^{\lfloor d / 2\rfloor}$ ) and the average space complexity $O\left(n^{\lfloor d / 2\rfloor}\right.$ ), over all insertion orders (uniformly).

This framework is only valid when the points are in general position, however. The most severe limitation is that when more than $d$ points belong to a facet, there is no bijection between the regions and the adjacency graph of the polytope. This information might be reconstructed if the polytope is quasi-simplicial. However even worse, when more than $d-1$ points belong to a ridge, there could be significantly more than $O\left(n^{\lfloor d / 2\rfloor}\right)$ free regions, up to $\Theta\left(n^{d}\right)$ perhaps. The formalism of Clarkson and Shor [12] is a little different in that a region consists of a single half-space and is in bijection with a facet. It does not a fortiori handle degenerate cases, and if a constant fraction of the points are on a single facet, the number of free regions is $\Theta\left(n^{d}\right)$. Therefore, these algorithms break down in the presence of degeneracies.

\subsection{Objects, Regions, and Conflicts}

In this section we present a new definition of objects, regions, and conflicts which, when applied to the formalism of influence graphs, yields an on-line algorithm for computing the $k$-dimensional convex hull of $n$ points in $\mathbb{R}^{d}$. The points can be in an arbitrarily degenerate position. The expected time complexity of this algorithm is $O\left(n \log n+n^{\lfloor k / 2\rfloor}\right)$ and its average space complexity $O\left(n^{\lfloor k / 2\rfloor}\right)$. The time needed to insert a point, averaged over all insertion orders of the points inserted so far (including the point itself), is $O\left(\log n+n^{\lfloor k / 2-1\rfloor}\right)$. Note that $k$ denotes the dimension of the convex hull and not the dimension $d$ of the ambient space.

As is done in [12], [5], and [4], we define the objects to be the points in $\mathbb{R}^{d}$. A region is defined as any ordered set of points in an affinely independent position. To refine the terminology, we define a $k$-region as a $(k+1)$-tuple $\left(p_{0}, \ldots, p_{k}\right)$ of points in an affinely independent position. In $\mathbb{R}^{d}$, at most $d+1$ points may be in an affinely independent position, thus a region is a $k$-region for some $k=0, \ldots, d$. Therefore, a region is determined by at most $d+1$ points, and a subset of $k$ objects determines at most $(k+1)$ ! regions, for any $0 \leq k \leq d$.

Our definition of conflict is recursive, based on the dimension. We denote by aff $(\mathcal{S})$ the affine hull of a point set $\mathcal{S}$. We say that a point $q$ conflicts with a $k$-region determined by $\left(p_{0}, \ldots, p_{k}\right)$ if one of the following conditions is fulfilled:

1. $q$ does not belong to the $k$-dimensional space aff $\left(p_{0}, \ldots, p_{k}\right)$, or 


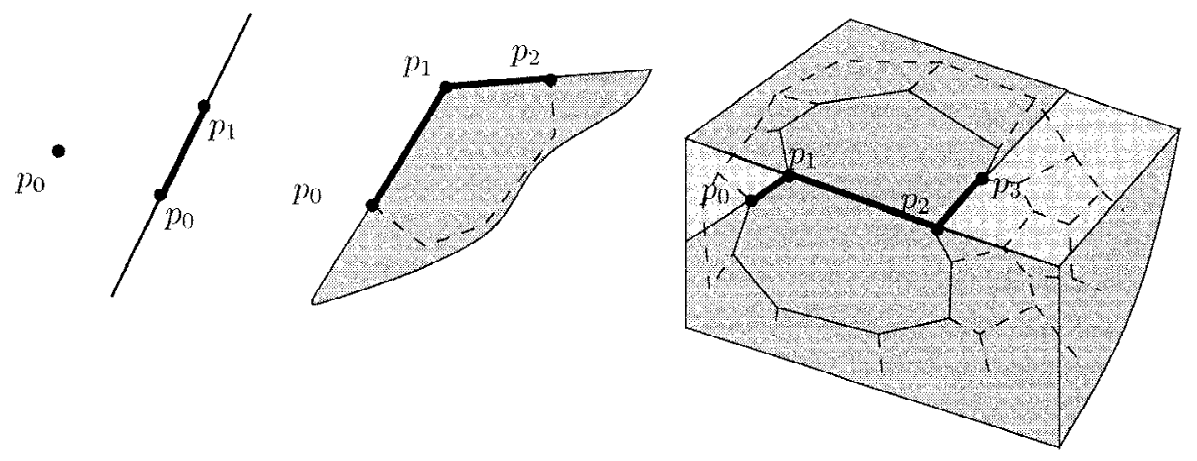

Fig. 1. The complement of the domain of influence is shown shaded in zero, one, two, and three dimensions.

2. $q$ and $p_{k}$ are on opposite sides of the hyperplane ${ }^{1}$ aff $\left(p_{0}, \ldots, p_{k-1}\right)$, or

3. $q$ and $p_{0}$ are on opposite sides of the hyperplane aff $\left(p_{1}, \ldots, p_{k}\right)$, or

4. if $q$ is on the hyperplane aff $\left(p_{0}, \ldots, p_{k-1}\right)$, then $q$ conflicts with the $(k-1)$-region determined by $\left(p_{0}, \ldots, p_{k-1}\right)$ in this hyperplane, or

5. if $q$ is on the hyperplane $\operatorname{aff}\left(p_{1}, \ldots, p_{k}\right)$, then $q$ conflicts with the $(k-1)$-region determined by $\left(p_{1}, \ldots, p_{k}\right)$ in this hyperplane.

The reader can check that this definition is equivalent to [4] and [5] if the set $\left\{q, p_{0}, \ldots, p_{k}\right\}$ is in general position. It introduces a refined conflict for points in a degenerate position, which extends the "flaps" of p. 242 of [13] to any dimension. Since the definition is recursive, we must define the notion of conflict with a 0-region. Following the convention that a region does not conflict with the points that determine it, we agree that a point $q$ that belongs to the zero-dimensional space that is the affine hull of a point $p_{0}$ does not conflict with the 0 -region determined by $p_{0}$, because $q$ and $p_{0}$ coincide.

This definition implies that two distinct points always conflict. A point conflicts with a 1-region (an oriented segment) if and only if either it does not lie on the line that supports the segment or else it is not contained in the closed segment. The domains of influence of a point, of a segment $\left(p_{0}, p_{1}\right)$, of a triangle $\left(p_{0}, p_{1}, p_{2}\right)$, and of a tetrahedron $\left(p_{0}, p_{1}, p_{2}, p_{3}\right)$ are illustrated in Fig. 1 . The shaded region is the complement of the domain of influence; in the three-dimensional case the portion that conflicts on the boundary of the wedge is nevertheless lightly shaded as if the shading of the inside of the wedge showed through. (Read also p. 242 of [13].) The reader is invited to picture the domain of influence of a $k$-region for a higher dimension $k$.

\subsection{How to Compute a Conflict Efficiently}

The on-line algorithms demand only to compute the orientation of $d+1$ points in $\mathbb{R}^{d}$, an operation that can be implemented using determinant sign computation. In the following,

\footnotetext{
${ }^{1}$ The notion of a hyperplane is relative to the $k$-dimensional flat spanned by $\left(p_{0}, \ldots, p_{k}\right)$, and the two (open) sides are half-spaces of dimension $k$ in this $k$-dimensional space.
} 
even though only points $p_{0}$ to $p_{k}$ are given by the region, we assume as an invariant that some points $p_{k+1}$ to $p_{d}$ are known such that $p_{0}, \ldots, p_{d}$ are affinely independent. How to maintain this invariant in our algorithm is explained in Section 3.1. Given $(k+1)$ points $p_{0}, \ldots, p_{k}$, if $p_{i}$ has homogeneous coordinates $\left(p_{i, j}\right)_{j=0, \ldots, d}$ with $p_{i, 0}>0$, we let the function $\operatorname{sign}\left(p_{0}, \ldots, p_{k}\right)$ compute the sign of the $(d+1)$-determinant of the matrix $\left(p_{i, j}\right)_{0 \leq i, j \leq d}$.

To test a conflict with a $k$-region using the straightforward recursive procedure suggested by the definition requires computing $\operatorname{sign}()$ of $k+1$ points in $\mathbb{R}^{d}$ at most $2^{k+1}$ times. Note that a test of conflict with $\left(p_{1}, \ldots, p_{k-1}\right)$ is common to tests of conflict with both $\left(p_{0}, \ldots, p_{k-1}\right)$ and $\left(p_{1}, \ldots, p_{k}\right)$, and using dynamic programming reduces the number of orientation tests to at most $k(k-1) / 2$. The following lemma shows that computing a conflict requires computing two orientation tests in the nondegenerate case, and at most $k+1$ such tests in any case

Lemma 1. Let $s_{\ell}=\operatorname{sign}\left(p_{0}, \ldots, p_{\ell-1}, q, p_{\ell+1}, \ldots, p_{k}\right)$ where $q$ is substituted for $p_{\ell}, 0 \leq \ell \leq k$. Then there is a value $j$ such that $s_{0}, s_{1}, \ldots, s_{j-1}=0$ and $s_{j} \neq 0$; similarly, there is a value $j^{\prime}$ such that $s_{j^{\prime}} \neq 0$ and $s_{j^{\prime}+1}, \ldots, s_{k-1}, s_{k}=0$. If $s_{j}=s_{j^{\prime}}=$ $\operatorname{sign}\left(p_{0}, \ldots, p_{k}\right)$, then $q$ does not conflict with the $k$-region $\left(p_{0}, \ldots, p_{k}\right)$, otherwise it does.

Proof. Note that all the $s_{\ell}$ 's cannot be null simultaneously since $p_{0}, \ldots, p_{k}$ are affinely independent. Thus there exist $j \leq j^{\prime}$ as in the lemma. The condition $s_{0}, s_{1}, \ldots, s_{j-1}=0$ and $s_{j^{\prime}+1}, \ldots, s_{k-1}, s_{k}=0$ shows that $q$ belongs to $\operatorname{aff}\left(p_{j}, \ldots, p_{j^{\prime}}\right)$. We may orient aff $\left(p_{j}, \ldots, p_{j^{\prime}}\right)$ by completing the basis $\left(p_{j}, \ldots, p_{j^{\prime}}\right)$, for instance, into $\left(p_{0}, \ldots, p_{d}\right)$. Thus $s_{j} \neq 0$ implies that $q$ does not belong to aff $\left(p_{j+1}, \ldots, p_{j^{\prime}}\right)$, and $s_{j^{\prime}} \neq 0$ implies that it does not belong to aff $\left(p_{j}, \ldots, p_{j^{\prime}-1}\right)$ either.

We examine first the conflict with region $\left(p_{j}, \ldots, p_{j^{\prime}}\right)$. Conditions 1,4 and 5 are not fulfilled by our previous observations. Condition 2 is equivalent to $s_{j}=\operatorname{sign}\left(p_{0}, \ldots, p_{k}\right)$ and condition 3 to $s_{j^{\prime}}=\operatorname{sign}\left(p_{0}, \ldots, p_{k}\right)$. Hence there is no conflict if and only if $s_{j}=s_{j^{\prime}}=\operatorname{sign}\left(p_{0}, \ldots, p_{k}\right)$. We call that condition $C$.

We can now finish the proof by a double induction on $\ell$ and $\ell^{\prime}$, showing that $q$ does not conflict with $\left(p_{\ell}, \ldots, p_{\ell^{\prime}}\right)$ if and only if $C$ is satisfied. Indeed, we just established the base case $\ell=j, \ell^{\prime}=j^{\prime}$. If $\ell<j, q$ belongs to $\operatorname{aff}\left(p_{j}, \ldots, p_{j^{\prime}}\right)$ and hence to aff $\left(p_{\ell+1}, \ldots, p_{\ell^{\prime}}\right)$, so condition 4 is equivalent to $C$. Similarly for condition 5 if $j^{\prime}<\ell^{\prime}$. Finally, for $\ell<j \leq j^{\prime}<\ell^{\prime}$, conditions 1-3 are never satisfied. The lemma follows by induction for $\ell=0$ and $\ell^{\prime}=k$.

\subsection{Regions Defined and Without Conflict}

Now we come to the characterization of the regions defined and without conflict over a set of points. A region is defined and without conflict over a set $\mathcal{S}$ of points if it determined by points in $\mathcal{S}$ and if it does not conflict with any point in $\mathcal{S}$. Recall that a region does not conflict with the points that determine it. For concision, when the set $\mathcal{S}$ is understood, we speak of a free region. 
The lemma below offers a characterization of regions defined and without conflict over a set of points.

Lemma 2. A region $\left(p_{0}, \ldots, p_{k}\right)$ is defined and without conflict over a set $\mathcal{S}$ of points in $\mathbb{R}^{d}$ if and only if aff $(\mathcal{S})$ has dimension $k$ and if, for any pair $(i, j), 0 \leq i<j \leq k$, the $(j-i)$ dimensional space aff $\left(p_{i}, \ldots, p_{j}\right)$ contains a unique $(j-i)$-face $F_{i, j}$ of $\operatorname{conv}(\mathcal{S})$, and $p_{i}, \ldots, p_{j}$ are vertices of $F_{i, j}$.

Proof. Observe that if there exists a $(j-i)$-face $F_{i, j}$ of $\operatorname{conv}(\mathcal{S})$, then necessarily $F_{i, j}=\operatorname{conv}(\mathcal{S}) \cap H_{i, j}$ where $H_{i, j}=\operatorname{aff}\left(p_{i}, \ldots, p_{j}\right)$. This proves the uniqueness.

First assume that a region $\left(p_{0}, \ldots, p_{d}\right)$ is defined and without conflict over $\mathcal{S}$. Then condition 1 implies that $\mathcal{S}$ is contained in $\operatorname{aff}\left(p_{0}, \ldots, p_{k}\right)$ and thus has dimension at most $k$. The dimension is $k$ because $p_{0}, \ldots, p_{k}$ are affinely independent. Hence $F_{0, k}=$ $\operatorname{conv}(\mathcal{S})$ and is a face of itself. Conditions 4 and 5 imply that $\left(p_{i}, \ldots, p_{j}\right)$ is a $(j-i)$ region defined and without conflict over $\mathcal{S} \cap H_{i, j}$. By condition 2, no point of $\mathcal{S} \cap H_{i, j}$ belongs to one side of the hyperplane $H_{i, j-1}$ in $H_{i, j}$ and so $H_{i, j-1}$ supports $F_{i, j-1}$ within $H_{i, j}$. This proves that $F_{i, j-1}$ is a facet of $F_{i, j}$. Similarly, $F_{i+1, j}$ is a facet of $F_{i, j}$. By induction on the dimension $j-i$, from $k$ down to 0 , we have shown that $F_{i, j}$ is a face of $\operatorname{conv}(\mathcal{S})$ for all $i \leq j$. Finally, this implies that $p_{\ell}=F_{\ell, \ell}$ is a vertex of $\operatorname{conv}(\mathcal{S})$ and thus of any $F_{i, j}$ for $i \leq \ell \leq j$.

Conversely, assume that $F_{i, j}$ is a $(j-i)$-face of $\operatorname{conv}(\mathcal{S})$ for all $i \leq j$. Then we will prove that $\left(p_{i}, \ldots, p_{j}\right)$ is defined and without conflict over $\mathcal{S} \cap H_{i, j}$. If we assume moreover that $\mathcal{S}$ has dimension $k$, then $\mathcal{S}$ is contained in $H_{0, k}$ and thus $\left(p_{0}, \ldots, p_{k}\right)$ is free over $\mathcal{S}$ which proves the lemma. The proof is by induction on $j-i$, but this time from 0 to $k$. If $j=i$, the induction hypothesis is trivially satisfied as the 0-region $\left(p_{i}\right)$ is defined and without conflict over $\left\{p_{i}\right\}=\mathcal{S} \cap H_{i, i}$. For $i<j$, let $q$ be any point of $\mathcal{S} \cap H_{i, j}$. Since $q \in H_{i, j}$, condition 1 is not satisfied. Since $F_{i, j-1}$ is a face of $F_{i, j}, q$ and $p_{j}$ cannot be on different sides of $H_{i, j-1}$ in $H_{i, j}$ which prevents condition 2. Similarly $q$ and $p_{i}$ cannot be on different sides of $H_{i+1, j}$ in $H_{i, j}$ which prevents condition 3. By induction, if $q$ belongs to $H_{i, j-1}$, then $q$ does not conflict with the region $\left(p_{i}, \ldots, p_{j-1}\right)$. Similarly, if $q$ belongs to $H_{i+1, j}$, then $q$ does not conflict with $\left(p_{i+1}, \ldots, p_{j}\right)$. Since $q$ does not fulfill any of the five conditions that define a conflict with $\left(p_{i}, \ldots, p_{j}\right)$, that region is free over $\mathcal{S} \cap H_{i, j}$.

In particular, the faces $\left(F_{0, j}\right)_{j=0, \ldots, k}$ form an ascending sequence of incident faces, i.e., a chain in the incidence graph. Moreover, $F_{i+1, j}$ can be obtained by pivoting from $F_{i, j-1}$ around $F_{i+1, j-1}$ inside $F_{i, j}$, and thus all the $F_{i, j}$ 's can be retrieved from the knowledge of the $F_{0, j}$ 's. This and the results of Section 4 show a bijection between the free regions, the maximal chains in the incidence graph, and the maximal-dimensional simplices in the barycentric triangulation, which is depicted in Fig. 2. The following theorem is an immediate consequence of those bijections and of Corollary 9.

Theorem 3. Let $\mathcal{S}$ be a set of $n$ points in $\mathbb{R}^{d}$, not necessarily in general position. If $\operatorname{conv}(\mathcal{S})$ is a $k$-polytope, $k \leq d$, then the number of regions defined and without conflict over $\mathcal{S}$ is $O\left(n^{\lfloor k / 2\rfloor}\right)$. 

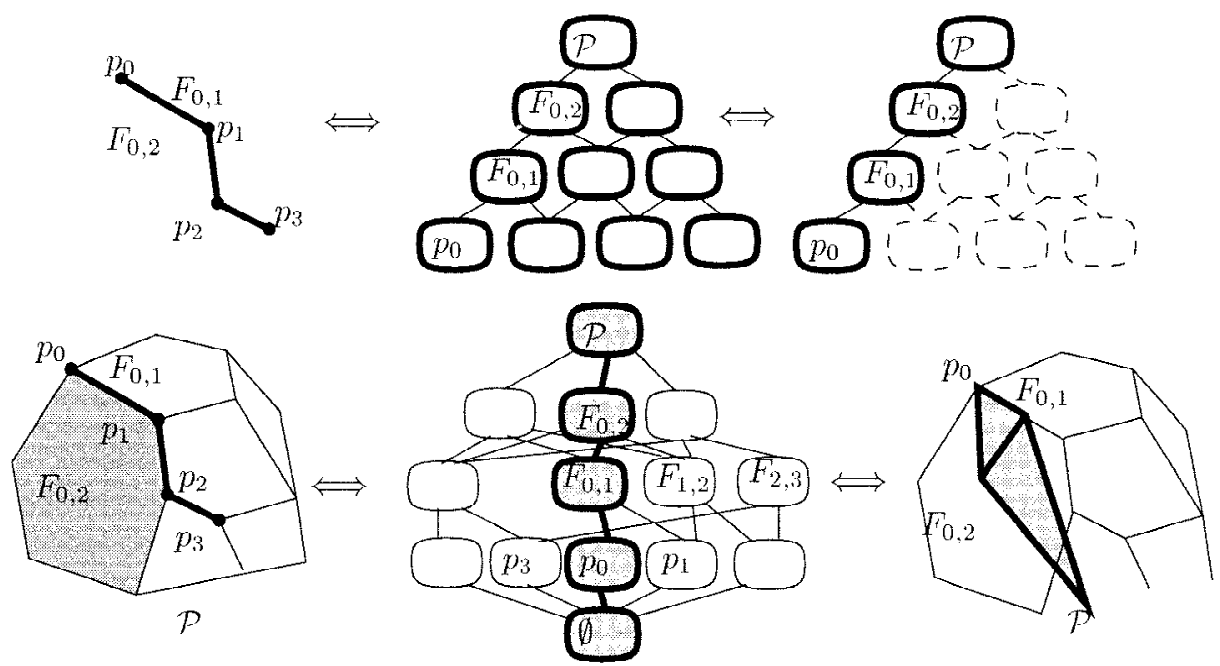

Fig. 2. Bijection between free regions, maximal chains in the incidence graph, and maximal-dimensional simplices in the barycentric triangulation. On the top, the bijection between the free region, the sublattice of the $F_{i, j}$ 's, and the chain of the $F_{0, j}$ 's.

If Conjecture 10 is true, then the upper bound can be sharpened to the complexity of the incidence graph of the polytope $\operatorname{conv}(\mathcal{S})$, an output-sensitive result for the number of regions.

\subsection{An Algorithm?}

We derived this formalism to adapt a randomized on-line algorithm for computing convex hulls. The algorithm would follow the general framework of the influence graph, but we would have to explain how to perform the update phase and satisfy the update conditions. In particular, we would have to explain how to derive the new regions from the free regions of $\mathcal{P}$ that are killed by $p_{n}$ and also how to set up the arcs of the influence graph so that each node has a bounded number of children and the inclusion property is verified. When the points are in general position, the free regions (and therefore the algorithm) are no different from those of [4] and [5].

Although the framework adapts nicely to degenerate cases, the number of free regions is still substantially higher than that of facets because we distinguish between all the permutations of the points defining the ridges. Thus the storage is also multiplied by the same proportions. At this point, we could explicit the on-line algorithm using the influence graph. However, in the next section we use such an algorithm as a reference for analyzing a simpler and better algorithm based on a different yet related data structure, which will store those regions implicitly. So we choose not to develop all the details of the influence graph algorithm. 


\section{Making the Incidence Graph Persistent}

In this section we present an algorithm that maintains the incidence graph of the polytope under the insertion of a new point, using the beneath-beyond method of Seidel (refer to [15] for a detailed account). In the beneath-beyond method, the points need to be inserted in lexicographic order, so as to find the conflicts efficiently. The influence graph allows us to find the conflicts while allowing a random order of insertion, but needs to keep the history and the full set of free regions, which is bigger than the incidence graph by at least a factor of $(k+1)$ !. Nevertheless, we show that we do not need an influence graph; instead, we propose to make the incidence graph persistent in order to store the history of the insertions. This allows us to randomize the order of insertion of the points in the beneath-beyond method. We then show that the theorem of the influence graph applied to the framework presented in the previous section can be used to analyze the persistent incidence graph.

\subsection{Overview of the Beneath-Beyond Method}

We assume that the reader is familiar with the beneath-beyond method as presented in [15]. We only recall the facts that are needed by the exposition of our algorithm. The points are inserted in lexicographical order. When adding a point $p \in \mathbb{R}^{k}$ to a $k$-polytope $\mathcal{P}=\operatorname{conv}(\mathcal{S})$, two cases can happen: the point may or may not belong to the affine span of the polytope. If not, the dimension of the polytope increases by one. This case is called a pyramidal update. Otherwise, the new point is inserted, which creates new faces and incidences. We describe both cases below.

Pyramidal Updates. To detect this case, the algorithm maintains a list of $k+1$ points $q_{0}, \ldots, q_{k}$ that belong to $\mathcal{S}$, and such that they are affinely independent and their $k$ dimensional affine hull contains $\mathcal{P}$. To this list is appended a list of $d-k$ points such that the whole list consists of $d+1$ points $q_{0}, \ldots, q_{d}$ of $\mathbb{R}^{d}$ in general position. Before any point is inserted, $k=-1$ and $q_{0}, \ldots, q_{d}$ can be chosen as any affine base of $\mathbb{R}^{d}$. When a new point $p$ is inserted, it belongs to the affine hull of the first $k+1$ points if all the $d-k$ determinants $\operatorname{det}\left(q_{0}, \ldots, q_{i-1}, p, q_{i+1}, \ldots, q_{d}\right), k<i \leq d$, are all zero. Otherwise, we have detected a pyramidal update. Then all but maybe one of the $q_{i}$ 's, $i>k$, do not belong to the affine span of $q_{0}, \ldots, q_{k}$ and $p$. So we can replace $q_{k+1}$ (up to a renumbering) by $p$ so that $q_{0} \ldots, q_{k+1}$ belong to $\mathcal{S}$, are affinely independent, and their $(\mathrm{k}+1)$-dimensional affine hull contains $\operatorname{conv}(\mathcal{P} \cup\{p\})=\mathcal{P}^{\prime}$.

As explained in [15], the incidence graph of $\mathcal{P}^{\prime}=\operatorname{conv}(\mathcal{P} \cup\{p\})$ consists of that of $\mathcal{P}$, plus a node $\hat{f}=\operatorname{conv}(f \cup\{p\})$ for each node $f$ of $\mathcal{P}$, and an arc from $f$ to $\hat{f}$.

Nonpyramidal Updates. When $p$ belongs to the affine span of $\mathcal{P}$, we describe the changes in the incidence graph using a color scheme for the faces [15]. A facet of $\mathcal{P}$ is colored yellow if their supporting hyperplane contains $p$, red if their supporting hyperplane separates $p$ and $\mathcal{P}$, and blue otherwise. Colors then bleed to the lowerdimensional faces, a face getting the fusion of the colors of the facets which contain it 
(red and blue making purple, red and yellow giving orange, blue and yellow yielding green, and all three together as brown).

We outline the changes in the incidence graph [15]. Blue, purple, brown faces and their incident arcs remain in the incidence graph. Red, orange, yellow faces and their incident arcs are not in the incidence graph of the new polytope $\mathcal{P}^{\prime}=\operatorname{conv}(\mathcal{P} \cup\{p\})$. A purple or brown face $f$ induces a new face $\hat{f}=\operatorname{conv}(f \cup\{p\})$ of dimension one higher, which contains $f$, and is incident to $f$ and to all $\hat{g}$ where $g$ ranges over all incident subfaces of $f$. A yellow face induces a new face $\bar{f}=\operatorname{conv}(f \cup\{p\})$ of the same dimension as $f$, which is incident to $\bar{g}$ or $g$ as appropriate, where $g$ ranges over all subfaces of $f$, and to $\hat{h}$ where $h$ ranges over all brown subfaces of $f$ incident to an orange incident subface of $f$. As explained in [15], the coloring can be computed by finding all the red facets. We call this the location phase. From the red facets, it is possible to access all nonblue facets and ridges, then recursively finding all the remaining nonblue faces in decreasing order of their dimension, then updating the arcs incident to the faces in decreasing order of their dimension. Since the points are inserted in lexicographic order, there must be a red facet incident to the last vertex, and hence the search for a red facet can be efficiently limited to the facets incident to the last vertex. Thus the location phase is easy in the beneath-beyond method.

\subsection{The Persistent Incidence Graph}

We introduce a persistent incidence graph as a data structure to maintain efficiently the incidence graph of a polytope under insertion of new vertices (see also [14] and [19] for other examples of persistent data structures). The persistent graph stores the sequence of polytopes $\mathcal{P}_{1}, \ldots, \mathcal{P}_{n}$ obtained incrementally by adding the points of a set $\mathcal{S}$ of $n$ points in $\mathbb{R}^{d}, p_{1}, p_{2}, \ldots, p_{n}$ in that order. Much like the incidence graph [5], [15], the persistent incidence graph consists of nodes representing the faces of the successive polytopes, and of arcs linking two incident faces. The arcs are oriented from lower to higher-dimensional faces, but can be accessed in both directions. As in the incidence graph, they will be followed from the empty face toward the higher dimension for the location phase of the algorithm, and in both directions once the faces are colored and in the update phase.

One can think of the persistent graph as the superposition of all the incidence graphs. In particular, a face or an arc stored in the persistent graph is a face or an arc of all the polytopes in a consecutive subsequence $\mathcal{P}_{i}, \ldots, \mathcal{P}_{j-1}$. We therefore associate with each arc a time interval $[i, j$ [ that begins at its creation date $i$ and ends just before its destruction date $j$. If the arc is in the incidence graph of the last polytope in the sequence, $\mathcal{P}_{n}$, its time interval is of the form [i, $\infty$ [. (This is to ensure that, when inserted in $p_{n+1}$, the arcs which are still in $\mathcal{P}_{n+1}$ have the same time interval and therefore do not have to be changed.) The nodes have incoming arcs coming from incident faces of lower dimension, and outgoing arcs to faces of higher dimension. We note that once a node is created, its incoming arcs will never change, because the geometry has determined what the subfaces of its corresponding face are. However, a face can be the subface of different faces in $\mathcal{P}_{i}$ and $\mathcal{P}_{j}, j>i$, so the outgoing arcs of its corresponding node can be modified in a way that will be described in the update phase below. 
To retrieve the graph of $\mathcal{P}_{i}$ from the persistent incidence graph can be done easily by starting at the empty face, and following the arcs whose time interval contains $i$ to access the faces in increasing order of their dimensions. In particular, $\mathcal{P}_{n}$ can be retrieved in time proportional to its complexity by looking at the outgoing arcs of each node in reverse order, starting at the last (the arcs are added to a node in chronological order).

In addition, each face of some $\mathcal{P}_{i}$ that has dimension $\operatorname{dim}\left(\mathcal{P}_{i}\right)-2$ (those faces are sometimes called ridges) stores a number of killer pointers. As is made clear in the analysis, killer pointers are strongly related to the arcs in the influence graph of the previous section. We will explain how to set and use these pointers in the algorithm. These pointers also have a time stamp, much like the time intervals for arcs, but it consists of a single number. This time stamp stores the date at which the pointer was set. The killer pointers are stored at each node by increasing order of their time stamps. We merely require that, for any point $p$ and any $j$, if a ridge of $\mathcal{P}_{j}$ is not blue with respect to $p$, then it is pointed to by a ridge of some $\mathcal{P}_{i}(i<j)$ which is not blue with respect to $p$ through a killer pointer whose time stamp belongs to $[i, j[$. We call this the inclusion property because it guarantees that the set of points with respect to which a ridge is colored red is included in the union of all the similar sets for the ridges whose killer pointers point to it. It is strongly related to the inclusion property of the influence graph.

\subsection{Handling Insertions}

We now detail the modifications to the beneath-beyond method that our algorithms must perform to operate on a persistent incidence graph when inserting a new point $p$ at stage $n$.

Pyramidal Updates. In addition to the copy-and-link procedure of the beneath-beyond method, the time stamps of the new arcs are set to $[n, \infty[$ and a killer pointer is added from each ridge $f$ of $\mathcal{P}$ to the corresponding ridge $\hat{f}$ of $\mathcal{P}^{\prime}$ with time stamp $n$. Note that this maintains the inclusion property for killer pointers as stated above.

Nonpyramidal Updates. When $p$ belongs to the affine span of $\mathcal{P}$, the faces that are not in the incidence graph of $\mathcal{P}^{\prime}$ and their incident arcs are said to be killed, and the algorithm performs a location phase to find the nodes that are killed. This phase is trivial in the beneath-beyond method [15] since we can start a traversal at the last inserted point, and it always yields a red facet.

Below, we explain how to find the red facets by using the persistent graph. It is now possible that $p$ lies inside $\mathcal{P}$. In that case, there are no red facets, $\mathcal{P}^{\prime}=\mathcal{P}$, and nothing else need be done. Otherwise, the algorithm performs the update phase as in the beneathbeyond method, creating the new nodes and arcs, and linking the new nodes between themselves and to the incidence graph of $\operatorname{conv}\left(\mathcal{P} \cup\left\{p_{n}\right\}\right)=\mathcal{P}^{\prime}$. The major modification is that we are careful not to remove the killed nodes and arcs from the incident graph. Instead, we set the upper bounds of their time interval to $n$. This ensures that they are not considered in the incidence graph of $\mathcal{P}^{\prime}$. When adding a point $p$, a new ridge is always the convex hull of $p_{n}$ and of a purple, green or brown $(d-3)$-face of $\mathcal{P}$. Since the set 
of purple or yellow faces is isomorphic to a $(d-1)$-polytope [15], this $(d-3)$-face is incident to exactly two purple or green ridges of $\mathcal{P}$. A killer pointer is added to link these two ridges to the new ridge of $\mathcal{P}^{\prime}$. It is easily checked that this satisfies the inclusion property for killer pointers [4]. This description can be carried over almost verbatim to the case of dimension 2 . We need only remember that every polytope has an empty face of dimension -1 , incident to all of its vertices.

We can now finish the description of the process that retrieves a red facet of $\mathcal{P}$. Simply follow the killer pointers in chronological order, starting with the empty face (that necessarily has one killer pointer to the vertices of the first two-dimensional polytope in the sequence). The color of each face is computed at the date set by the incoming killer pointer (it is the fusion of the colors of the two incidents facets at that date), and the search is recursively propagated through nodes with a red component. Because of the inclusion property, all the nonblue ridges of the last polytope $\mathcal{P}=\mathcal{P}_{n}$ are discovered in this traversal. Then the other steps of the beneath-beyond method are performed as outlined above (and in [15]), not forgetting to update the killer pointers as described above.

\subsection{Analysis}

Analyzing this algorithm would be a difficult task to perform directly. Fortunately, the persistent incidence graph is a condensed version of a (fictitious) influence graph as outlined in the previous section [4], [5]. It contains all the chains implicitly, therefore all the free regions as defined in the previous section. Indeed, let $F$ be a ridge of $\mathcal{P}_{i}$, incident to two facets of $\mathcal{P}_{i}$. One can always choose vertices $p_{0}, \ldots, p_{k-1}$ of $F$ and $p_{0}, p_{k}$ on the two incident facets, such that the region $\left(p_{0}, \ldots, p_{k}\right)$ is free over $\mathcal{S}_{i}$. (There are many ways to choose them, for instance, by choosing an ascending chain of faces that contains $F$ and using the bijections of the previous section.) Therefore:

Lemma 4. For each $i$, there is an injection from the pairs $(F, i)$ where $F$ is a ridge of $\mathcal{P}_{i}$ into the regions defined and without conflict over $\mathcal{S}_{i}$. Moreover, if we follow a killer pointer to $(F, i)$ when inserting $p$, then $p$ conflicts with the region associated with $(F, i)$.

The second part follows from the consideration that we only follow a pointer to $(F, i)$ if the node $F$ has a red component, hence one of the two incident facets must be red, which is enough to guarantee conflict.

The time to find nonblue faces is proportional to the number of nonblue faces of all the polytopes $\mathcal{P}_{1}, \ldots, \mathcal{P}_{n}$. By the lemma above, these faces are contained in the regions that would be traversed in the influence graph to find the free regions killed by the new point. The killer pointers only access the nonblue ridges of $\mathcal{P}$, which is always less than all the free regions of $\mathcal{P}_{n}$ that conflict with $p$. Nevertheless, those nonblue ridges are enough to color all the faces of $\mathcal{P}$ as explained in [15].

The time necessary to update the persistent incidence graph is bounded by the number of nonblue faces of $\mathcal{P}_{n}$ and new faces of $\mathcal{P}^{\prime}$ [15]. We note that each such node has at most $d-1$ killer pointers leaving it, which is the analogue of the bounded number of children in the incidence graph. Each such node belongs to at least one region of the influence 
graph, and their number is clearly upper-bounded by the number of killed or new regions. Therefore the number of operations performed by the algorithm is upper-bounded by the complexity of a (fictitious) algorithm that uses an influence graph and verifies the update condition. The theorem of the influence graph and the results of Theorem 3 on the number of regions show:

Theorem 5. Let $d$ be a fixed constant. The persistent incidence graph maintains the convex hull of any set of $n$ points in $\mathbb{R}^{d}$ in expected time $O\left(n \log n+n^{\lfloor k / 2\rfloor}\right)$ and average space $O\left(n^{\lfloor k / 2\rfloor}\right)$, averaged over all possible insertion orders. The expected time needed to insert any point, averaged over all insertion orders of the points inserted so far (including the point itself), is $O\left(\log n+n^{\lfloor k / 2-1\rfloor}\right)$. Here $k$ denotes the dimension of the convex hull of the points.

\section{Triangulations of $d$-Polytopes}

Triangulation schemes come up naturally in the analysis of the previous algorithms, and also in the analysis of other pivoting algorithms [18], so it may be useful to study them for their own sake. Although the bounds are known in the worst case, we prove slightly tighter bounds and elucidate a few points, for instance, why a so-called bottom-vertex triangulation is not always a triangulation.

\subsection{Face-to-Vertex Subdivisions}

Here we denote by $\mathcal{F}(\mathcal{P})$ the set of faces of a polytope $\mathcal{P}$, and by $\mathcal{F}_{k}(\mathcal{P})$ the set of its $k$-faces. In particular, $\mathcal{F}_{0}(\mathcal{P})$ is the set of vertices of $\mathcal{P}$. A face-to-vertex map for the polytope $\mathcal{P}$ is a map $\pi$ from the set $\mathcal{F}(\mathcal{P})$ of faces of $\mathcal{P}$ onto the set $\mathcal{F}_{0}(\mathcal{P})$ of its vertices that maps each face to one of its vertices. Given such a map $\pi$, the face-to-vertex subdivision $\mathcal{T}_{\pi}(\mathcal{P})$ of $\mathcal{P}$ is a set of $d$-simplices obtained by recursively building the face-to-vertex subdivision of all the facets $f$ of $\mathcal{P}$ that do not contain $\pi(\mathcal{P})$, forming a set $\bigcup_{f} \mathcal{T}_{\pi}(f)$ of simplices, and lifting all these simplices toward $\pi(\mathcal{P})$. In other words, for each simplex $\sigma$ in $\bigcup_{f} \mathcal{T}_{\pi}(f)$, we insert $\operatorname{conv}(\sigma \cup\{\pi(\mathcal{P})\})$ into $\mathcal{T}_{\pi}(\mathcal{P})$. It is clear that each simplex in $\mathcal{T}_{\pi}(\mathcal{P})$ is $d$-dimensional. The number $t_{\pi}^{(d)}(\mathcal{P})$ of $d$-simplices in the face-to-vertex subdivision of $\mathcal{P}$ verifies $t_{\pi}^{(0)}(\mathcal{P})=1$ if $\mathcal{P}$ is a 0 -polytope, and, if $\mathcal{P}$ is a $d$-polytope,

$$
t_{\pi}^{(d)}(\mathcal{P})=\sum_{\substack{f \in \mathcal{F}_{d-1}(\mathcal{P}) \\ f \not \pi(\mathcal{P})}} t_{\pi}^{(d-1)}(f),
$$

where $f$ ranges over all the facets of $\mathcal{P}$ that do not contain the vertex $\pi(\mathcal{P})$. For simplicity, we write $t_{\pi}(\mathcal{P})$ for $t_{\pi}^{(d)}(\mathcal{P})$ when $\mathcal{P}$ is a $d$-polytope.

One may think that the face-to-vertex subdivision is a triangulation, but this is not always so. It is a triangulation if and only if, for any faces $f$ and $g$, if $f$ contains $g$, $g$ is not a simplex, and $\pi(f)$ is in $g$, then $\pi(g)=\pi(f)$. A sufficient condition is given by the slightly stronger compatibility condition: for any faces $f$ and $g$, if $f$ contains $g$ and $\pi(f)$ is in $g$, then $\pi(g)=\pi(f)$. The usual bottom-vertex triangulation 
(where the bottom vertex of each face is its geometrically lexicographically minimum vertex, given a system of Cartesian coordinates) satisfies this condition. In fact, the following lemma characterizes facet-to-vertex maps that satisfy the compatibility condition.

Lemma 6. Given any total ordering of the vertices, the map which sends any face to the minimum vertex it contains satisfies the compatibility condition. Conversely, any map that verifies the compatibility condition defines such a total ordering for the vertices.

Proof. Given a vertex ordering, construct $\pi$ as follows: for each face $f$ we let $\pi(f)$ be the smallest vertex of $f$ for this ordering. If $f$ contains $g$, and $v=\pi(f)$ is a vertex of $g$, then $v$ is smaller (by construction) than any vertex of $f$, therefore of $g$. Thus $\pi(g)=v$ and $\pi$ verifies the compatibility condition.

Let us now be given a face-to-vertex map $\pi$ that satisfies the compatibility condition. We now construct from $\pi$ a total order on the vertices of $\mathcal{P}$ such that the first vertex of any face $f$ is $\pi(f)$. The first vertex is naturally $\pi(\mathcal{P})$. Then we collect all the facets and enumerate their bottom vertices if it is not $\pi(\mathcal{P})$; the order in which these facets are processed does not matter, because the compatibility condition implies that if two facets $f_{1}$ and $f_{2}$ both contain $\pi\left(f_{1}\right)$ and $\pi\left(f_{2}\right)$, then $\pi\left(f_{1}\right)$ is the same as $\pi\left(f_{2}\right)$ as well as $\pi(g)$ for any face $g$ contained in $f_{1} \cap f_{2}$. We then process the $k$-faces similarly for decreasing dimension $k \leq d-2$. We can now prove that the first vertex in this enumeration process for any $k$-face $f$ is $\pi(f)$ by induction on $k$ starting at $k=d$ (trivial) and decreasing: either $\pi(f)$ is listed for a superface $h$ of $f$, so that it is listed (by induction) before any vertex of $h$ and hence of $f$, or else no vertex of $f$ has been listed before and therefore $\pi(f)$ is the first and only vertex of $f$ listed before we process the $(k-1)$-faces.

That any face-to-vertex subdivision of a $d$-polytope with $n$ facets has at most $O\left(n^{\lfloor d / 2\rfloor}\right)$ $d$-simplices was proved in [10] for the more general case of line-free polyhedra, by pushing the facets, a technique dual to pulling the vertices [17]. The careful reader will check that the counting argument used in [10] applies to subdivisions which are not triangulations. The dual statement seems to be part of the folklore, but it applies only to compatible maps, and hence to triangulations. The triangulation can be constructed by pulling the vertices in the corresponding order. We can also prove the following extension, whose proof we give after Theorem 8 .

Theorem 7. Any face-to-vertex subdivision (not necessarily a triangulation) of a polytope with $n$ vertices has at most $O\left(n^{\lfloor d / 2\rfloor}\right)$ simplices.

\subsection{Barycentric Triangulation}

Assume we have computed the center of gravity of $\mathcal{P}$, called the barycenter, and also that of all its faces. The barycentric triangulation is obtained by recursively triangulating all the facets of $\mathcal{P}$ (with respect to their barycenters) and lifting all these simplices toward the barycenter of the polytope. The number $t_{\mathrm{b}}^{(d)}(\mathcal{P})$ of $d$-simplices in the barycentric 
triangulation of a $d$-polytope $\mathcal{P}$ satisfies $t_{\mathrm{b}}^{(0)}(\mathcal{P})=1$ if $\mathcal{P}$ is a 0 -polytope, and

$$
t_{\mathrm{b}}^{(d)}(\mathcal{P})=\sum_{f \in \mathcal{F}_{d-1}(\mathcal{P})} t_{\mathrm{b}}^{(d-1)}(f)
$$

if $\mathcal{P}$ is a $d$-polytope, where $f$ ranges over all the facets of $\mathcal{P}$. For simplicity, we write $t_{\mathrm{b}}(\mathcal{P})$ instead of $t_{\mathrm{b}}^{(d)}(\mathcal{P})$ when $\mathcal{P}$ is a $d$-polytope. These numbers are preserved by duality.

Barycentric triangulations have been advocated by Brisson [6] as a uniform representation for structured manifolds. In the case of polytopes, it is easy to prove by a perturbation argument that $t_{\mathrm{b}}(\mathcal{P})=O\left(n^{\lfloor d / 2\rfloor}\right)$ for any $d$-polytope $\mathcal{P}$. Indeed $t_{\mathrm{b}}(\mathcal{P})=$ $O\left(n^{\lfloor d / 2\rfloor}\right)$ is maximized for simplicial polytopes (simply pull the vertices), and in that case $t_{\mathrm{b}}(\mathcal{P})=O\left(n^{\lfloor d / 2\rfloor}\right)$. The same argument does not apply to Theorem 7 except if the compatibility condition is true. The following theorem relates the sizes of barycentric triangulations and face-to-vertex subdivisions, and gives a finer bound on the size of a barycentric triangulation for a given $d$-polytope than the one obtained by the perturbation argument.

Theorem 8. For any d-polytope $\mathcal{P}$ and any map $\pi$ from $\mathcal{F}(\mathcal{P})$ onto $\mathcal{F}_{0}(\mathcal{P})$, we have

$$
t_{\pi}(\mathcal{P}) \leq t_{\mathrm{b}}(\mathcal{P})
$$

Conversely, there exists a face-to-vertex map $\pi$ such that

$$
t_{\mathrm{b}}(\mathcal{P}) \leq(d+1) ! t_{\pi}(\mathcal{P}) .
$$

The factor $(d+1)$ ! cannot be improved, as it is tight for a $d$-simplex.

Proof of Theorem 7. Let $\overline{\mathcal{P}}$ denote the dual of $\mathcal{P}$, so that $\overline{\mathcal{P}}$ has $n$ facets. By duality and Theorem 8 , there is a face-to-vertex map $\pi^{\prime}$ such that

$$
t_{\mathrm{b}}(\mathcal{P})=t_{\mathrm{b}}(\overline{\mathcal{P}}) \leq(d+1) ! t_{c\left(\pi^{\prime}\right)}(\overline{\mathcal{P}})=O\left(n^{d / 2}\right),
$$

where the last bound follows from Clarkson's result mentioned before Theorem 7 [10] and the fact that $\overline{\mathcal{P}}$ has $n$ facets.

Proof of Theorem 8 . The first inequality, namely, $t_{\pi}(\mathcal{P}) \leq t_{\mathrm{b}}(\mathcal{P})$, follows from the fact that $\mathcal{T}_{\pi}(\mathcal{P})$ can be obtained from $\mathcal{T}_{\mathrm{b}}(\mathcal{P})$ by dragging the barycenters of the faces toward their bottom vertex. Clearly, this process may collapse some simplices, but never creates other simplices, and hence the number of $d$-simplices in any face-to-vertex subdivision is less than the corresponding number in the barycentric triangulation. We call such a process a dragging process.

We now prove the second part of the theorem. More precisely, we prove that it is possible to choose $(d+1)$ ! face-to-vertex maps $\pi_{j}$ such that no $d$-simplex is collapsed during all the corresponding $(d+1)$ ! dragging processes. Therefore, we have

$$
t_{\mathrm{b}}(\mathcal{P}) \leq \sum_{j=0}^{(d+1) !} t_{\pi_{j}}(\mathcal{P}) \leq(d+1) ! t_{\pi}(\mathcal{P}),
$$

where $\pi$ is the $\pi_{j}$ that maximizes $t_{\pi_{j}}(\mathcal{P})$. 
We show how to construct the maps $\pi_{j}$. For each $k=0, \ldots, d$, and each $k$-face $f$ of $\mathcal{P}$, pick $k+1$ affinely independent vertices of $f$ and number them $p_{0}(f), \ldots, p_{k}(f)$.

Each $\pi_{j}$ is entirely determined by a sequence $\left(j_{0}, j_{1}, \ldots, j_{d}\right)$, where $j_{k}$ is comprised between 0 and $k$, by setting $\pi_{j}(f)=p_{j_{k}}(f)$ for any face $f$ whose dimension is $k$. There are exactly $(d+1)$ ! choices for $\pi_{j}$. The proof that no $d$-simplex can collapse during all the corresponding dragging processes follows by induction on $k$ of the fact that no $k$-simplex can be collapsed during all the corresponding dragging processes. Indeed, this is true for $k=0$ and $k=1$ (the midpoint of a segment cannot be dragged to both vertices at once). We note that a $k$-simplex in a $k$-face $f$ is the convex hull of the barycenter of $f$ and of a $(k-1)$-simplex $\Delta$ in a facet of $f$. By induction, this simplex is not collapsed for some choice of $j_{0}, j_{1}, \ldots, j_{k-1}$. The facet of $f$ containing $\Delta$ has dimension $k-1$, so it cannot contain simultaneously all the vertices $p_{0}(f), \ldots, p_{k}(f)$; for at least one choice of $j_{k}$, the simplex $\operatorname{conv}\left(p_{j_{k}}(f) \cup \Delta\right)$ does not collapse. The proof is complete by induction.

There is a well-known bijection between barycentric triangulations and chains in the incidence lattice [3], that implies that the number of chains in the lattice of a $d$ polytope is also $O\left(n^{\lfloor d / 2\rfloor}\right)$. This bijection maps the $d$-simplices in the barycentric triangulation of a polytope $\mathcal{P}$ to the maximal chains in the incidence lattice of $\mathcal{P}$, which are sometimes called flags [3]. Indeed, each $d$-simplex is, by construction, the convex hull of the centers of gravity of the $d$-face, of a facet, of a $(d-2)$-face, etc., until it all comes down to a vertex which is its own barycenter. This succession of facets gives a chain in the incidence graph that uniquely corresponds to the $d$-simplex. This bijection extends between all the simplices in the barycentric triangulation and the (possibly not maximal) chains in the incidence graph. The bijection is depicted in Fig. 3.

This bijection shows that there are as many $d$-simplices in the barycentric triangulation of a polytope as in that of its dual, because the dual incidence graph is the same with arcs reversed. With the previous theorem, we have thus proven that the number of monotone chains in the incidence graph of any polytope is $O\left(n^{\lfloor d / 2\rfloor}\right)$. This corollary is not new [3], [6].
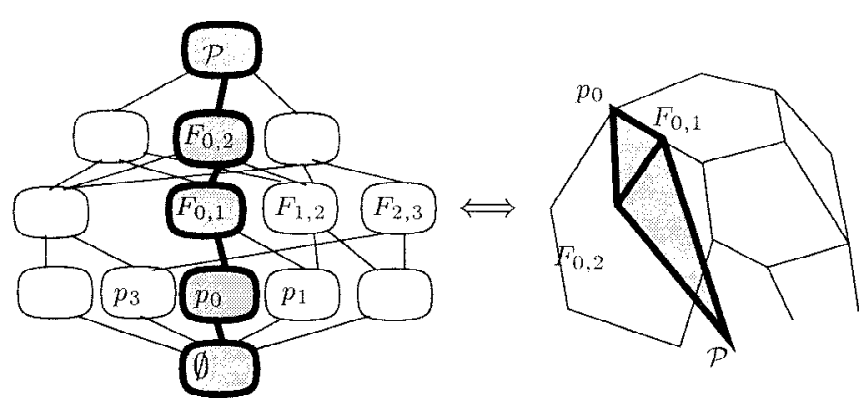

Fig. 3. Bijection between chains in the incidence graph and simplices in the barycentric triangulation. $F_{0,1}$ is a segment on the edge $p_{0}, p_{1}$ and $F_{0,2}$ a triangle on the face $p_{0}, p_{1}, p_{2}$. 
Corollary 9. The barycentric triangulation of a d-polytope $\mathcal{P}$ has $O\left(n^{\lfloor d / 2\rfloor}\right)$ $d$-simplices, where $n$ is either the number of vertices or the number of facets of $\mathcal{P}$. There is a bijection between the simplices of this triangulation and the ascending chains in the incidence graph.

\subsection{Output-Sensitivity}

The complexities of the subdivisions above all follow from the upper bound theorem [15], [17]. They do not, however, take into account the actual complexity of the incidence graph of the polytope. In view of the results above, we give the following conjecture, already stated in [21] in a slightly different form:

Conjecture 10. For a fixed dimension d, the number of simplices in any face-to-vertex or barycentric subdivision of a polytope, hence the number of maximal chains in the incidence graph of a polytope, is at most proportional to the complexity of the incidence graph.

As observed by Raimund Seidel (private communication), the constant of proportionality must depend superexponentially on $d$. For instance, a $d$-dimensional cube has $2 d$ facets, $2^{d}$ vertices, and $3^{d}$ faces of all dimensions; the number of incidences between these faces is $O\left(d 3^{d}\right)$, since the cube is a simple polytope. Yet, any triangulation of its boundary requires at least $(d / 2)^{d / 2}(d-1)$-simplices, which is superexponentially larger.

We now give some support to the conjecture. As mentioned in [21] (and reference [8] by Billera and Lee therein), the conjecture is verified for simple and simplicial polytopes in any dimension $d$. Simplicial polytopes are those whose faces are all simplices, and simple polytopes are their dual counterparts. In these cases, the number of chains is even more precisely $\Theta\left(d^{\lfloor d / 2\rfloor}\left(n_{0}+n_{d-1}\right)\right)$, where $n_{k}$ is the number of $k$-faces for any $k$.

Quasi-simplicial polytopes are those whose faces are all simplicial, except maybe the facets. The number of chains in a quasi-simplicial $d$-polytope is $(d-1)$ ! times the number of chains that link a $(d-2)$-face to the $d$-face of the polytope; this number is exactly twice the number $n_{d-2}$ of $(d-2)$ faces, and so there are $2(d-1) ! n_{d-2}$ chains in a quasi-simplicial $d$-polytope.

It is easy to verify the conjecture for $d$-polytopes, with any $d \leq 3$. As argued by Raimund Seidel (private communication), it is still valid for 4-polytopes. Indeed, the 2-faces are contained in exactly two 3-faces of the 4-polytope, and in the unique 4-face. Dually, the 1 -faces contain exactly two 0 -faces, contained in the unique empty face. Thus, if $n_{1,2}$ is the number of incidences between the 1-faces and the 2-faces, there are exactly $4 n_{1,2}$ chains in the incidence graph, hence 4 -simplices in the barycentric triangulation.

For 5-polytopes, the number of chains is also easily shown to be exactly equal to four times the number of chains from any 1-face to any 3-face. Denoting by $\operatorname{sub}(f)$ and $\sup (f)$ the numbers of sub- and superfaces of a face $f$ of a polytope $\mathcal{P}$, this latter number 
equals the "second moment" sum

$$
K_{2}(\mathcal{P})=\sum_{f \in \mathcal{F}_{2}(\mathcal{P})} \operatorname{sub}(f) \sup (f) .
$$

It is known that this parameter $K_{2}(\mathcal{P})$ is maximized for cyclic polytopes [1], for which it equals $12 n_{3}(\mathcal{P})$. Nevertheless, it is not known if this parameter $K_{2}$ is linear in the complexity of the incidence graph of any 5-polytope $\mathcal{P}$.

The conjecture is still open (as far as we know) for general $d$-polytopes, with a fixed $d \geq 5$.

\section{Conclusion and Bibliographic Notes}

We have given an on-line algorithm that maintains the convex hull or the Voronoi diagram of a set of points in any dimension, which works and is worst-case optimal even if the points are in degenerate position. A possible extension is to make the algorithm fully dynamic (deletions as well as insertions). Hopefully, this should not be very hard by using the paradigm presented in [5] and [22].

Often, the limiting factor in enumerating facets of high-dimensional polytopes is the storage [1]. Our data structure, like its analogues, allows us to locate new conflicts rapidly at the expense of storing a huge graph. There are other candidates for location structures, with various tradeoffs between storage and location time; one could, for instance, select a random subset of $\sqrt{n}$ of the vertices and walk from there toward the new point, etc. Our method is limited to a domain where $d$ is a fixed, small constant. Nevertheless, computing three-dimensional Voronoi diagrams, power diagrams, and Delaunay tessellations is equivalent to computing four-dimensional convex hulls. To diminish the storage, Swart [21] has shown how to abbreviate the incidence graph to take into account the nonsimplicial faces only. We suspect that the persistent incidence graph could also be abbreviated, although we have not carried the analysis through.

It has also been shown in [21] and [18] how the running times of several algorithms depend on the parameter $K_{2}$ introduced in Section 4.3, and on the number of monotone chains in the abbreviated incidence graph. The bounds we supply here prove partially the conjecture stated in [21], in that the number of chains in the abbreviated incidence graph of a polytope with $n$ vertices and $f$ facets is $\gamma(n, f)=O_{d}\left(n^{\lfloor d / 2\rfloor}\right)$ for fixed $d$ (in the terminology used there). This turns out to provide a bound to the complexity of several pivoting algorithms [21], [18]. Unfortunately, we cannot prove the full conjecture in dimensions higher than 4 and we ask again: for a fixed $d \geq 5$, is the number of chains in the incidence graph of a $d$-polytope proportional to the complexity of the incidence graph? Answering this conjecture would provide some insight on the complexity of linear programming and facet enumeration. Note that it would not affect the output-sensitivity of our algorithms since incremental algorithms cannot run in time that depends solely on the complexity of the final lattice [1].

Finally, the problem that spurred this investigation is that of degeneracies. Is there a natural extension of the formalism which may define a degenerate case for arbitrary objects and regions (e.g., two distinct regions have the same domain of influence) and a generic algorithm to handle these degeneracies? We do not know. 


\section{References}

1. D . Avis, D. Bremner, and R. Seidel. How good are convex hull algorithms? Comput. Geom. Theory Appl., 7:265-302, 1997.

2. D. Avis and K. Fukuda. A pivoting algorithm for convex hulls and vertex enumeration of arrangements and polyhedra. Discrete Comput. Geom., 8:295-313, 1992.

3. L. Billera and A. Björner. Face numbers of polytopes and complexes. In The Handbook of Discrete and Computational Geometry, J. E. Goodman and J. O'Rourke, eds., pages 251-310, CRC Press, Boca Raton, FL, 1997.

4. J.-D. Boissonnat, O. Devillers, R. Schott, M. Teillaud, and M. Yvinec. Applications of random sampling to on-line algorithms in computational geometry. Discrete Comput. Geom., 8:51-71, 1992.

5. J.-D. Boissonnat and M. Yvinec. Algorithmic Geometry. Cambridge University Press, Cambridge, 1998. Also in French: Géométrie Algorithmique, Ediscience International, Paris, 1995.

6. E. Brisson. Representing geometric structures in $d$ dimensions: topology and order. In Proc. 5th Ann. ACM Symp. Comput. Geom., pages 218-227, 1989.

7. C. Burnikel, K. Mehlhorn, and S. Schirra. On degeneracy in geometric computations. In Proc. 5th ACMSIAM Symp. Discrete Algorithms, pp. 16-23, 1994.

8. D. R. Chand and S. S. Kapur. An algorithm for convex polytopes. J. Assoc. Comput. Mach., 17:78-86, 1970 .

9. B. Chazelle. An optimal convex hull algorithm in any fixed dimension. Discrete Comput. Geom., 10:377409, 1993.

10. K. L. Clarkson. A randomized algorithm for closest-point queries. SIAM J. Comput., 17:830-847, 1988.

11. K. L. Clarkson, K. Mehlhorn, and R. Seidel. Four results on randomized incremental constructions. Comput. Geom. Theory Appl., 3(4):185-212, 1993.

12. K. L. Clarkson and P. W. Shor. Applications of random sampling in computational geometry, II. Discrete Comput. Geom., 4:387-421, 1989.

13. M. de Berg, M. van Kreveld, M. Overmars, and O. Schwarzkopf. Computational Geometry: Algorithms and Applications. Springer-Verlag, Berlin, 1997.

14. J. R. Driscoll, N. Sarnak, D. D. Sleator, and R. E. Tarjan. Making data structures persistent. J. Comput. System Sci., 38:86-124, 1989.

15. H. Edelsbrunner. Algorithms in Combinatorial Geometry. Volume 10 of EATCS Monographs on Theoretical Computer Science. Springer-Verlag, Heidelberg, 1987.

16. I. Emiris. A Complete Implementation for Computing General Dimensional Convex Hulls. Research Report 2551, INRIA, 1996.

17. B. Grünbaum. Convex Polytopes. Wiley, New York, 1967.

18. G. Rote. Degenerate convex hulls in high dimensions without extra storage. In Proc. 8th Ann. ACM Symp. Comput. Geom., pages 26-32, 1992.

19. N. Sarnak and R. E. Tarjan. Planar point location using persistent search trees. Commun. ACM, 29:669-679, 1986.

20. R. Seidel. Output-Size Sensitive Algorithms for Constructive Problems in Computational Geometry. Ph. D. Thesis. Technical Report TR 86-784, Department of Computer Science, Cornell University, Ithaca, NY, 1986.

21. G. F. Swart. Finding the convex hull facet by facet. J. Algorithms, 6:17-48, 1985.

22. M. Teillaud. Towards Dynamic Randomized Algorithms in Computational Geometry. Volume 758 of Lecture Notes Computer Science. Springer-Verlag, Berlin, 1993.

Received May 30, 1998, and in revised form March 26, 1999. 